\title{
Resposta superovulatória de caprinos da raça Boer submetidos a produtos com diferentes relações FSH:LH
}

Superovulatory response of Boer goat products submitted to different relationships with FSH:LH

\author{
CHALHOUB, Marcos ${ }^{1 *}$; PORTELA, Ana Paula Mota ${ }^{1}$; FEITOSA, Leandro Moreira \\ de Castro ${ }^{1}$; ARAUJO, Endrigo Adonis Braga ${ }^{1}$; OLIVEIRA, Sidnei Nunes de ${ }^{1}$; \\ RODRIGUES, Alexandra Soares ${ }^{1}$; LOIOLA, Marcus Vinícius Galvão ${ }^{1}$; ANDRADE, \\ Bruno Henrique de Araújo ${ }^{1}$; FERRAZ, Priscila Assis ${ }^{1}$; RIBEIRO FILHO, Antonio \\ de Lisboa $^{1}$
}

\footnotetext{
${ }^{1}$ Universidade Federal da Bahia, Escola de Medicina Veterinária e Zootecnia, Departamento de Anatomia, Patologia e Clínicas Veterinárias, Salvador, Bahia, Brasil.

*Endereço para correspondência: chalhoub@ufba.br
}

\section{RESUMO}

Objetivou-se averiguar a eficiência e padrões na resposta superovulatória de caprinos da raça Boer submetidos a produtos com diferentes relações FSH:LH. Foram utilizadas 43 fêmeas caprinas da raça Boer. Em um dia aleatório do ciclo estral, os animais receberam metade de um implante de progesterona $\left(\mathrm{Crestar}^{\circledR}\right)$. No dia 11 , iniciou-se o estímulo à superovulação por meio da utilização do FSH fracionado em seis doses decrescentes administradas a cada 12 horas e os animais foram separados em dois grupos: o grupo I (GI, n=22) recebeu $200 \mathrm{mg}$ de FSH (Folltropin ${ }^{\circledR}$ ) por via intramuscular (i.m.) nos dias D11 (62,5mg), D12 (35,0mg) e D13 $(2,5 \mathrm{mg})$, enquanto o grupo II (GII, $\mathrm{n}=21)$ recebeu 300UI de FSH (Pluset ${ }^{\circledR}$ ) por via i.m. nos dias D11 (75UI), D12 (50UI) e D13 (25UI). Nas 60 e 72h após o início da superovulação foram retirados os implantes, e aplicaram-se duas doses de $125 \mu \mathrm{g}$ de Cloprostenol (Ciosin ${ }^{\circledR}$ ) por via intravulvosubmucosa em todos os animais. Doze, 24 e 36 horas após a remoção dos implantes, as fêmeas foram colocadas com quatro reprodutores para serem cobertas. No D16, os implantes foram reinseridos e, nas $24 \mathrm{~h}$ antes da coleta dos embriões, retiraram-se os implantes, e administrou-se $125 \mu \mathrm{g}$ de Cloprostenol i.m. a cada animal. Os embriões foram coletados no D21, selecionados e classificados. Os resultados obtidos nesse estudo mostraram que não houve diferença estatística entre os grupos, quanto ao número de estruturas recuperadas, embriões viáveis, embriões degenerados e estruturas não fertilizadas. Portanto, as diferentes relações FSH:LH avaliadas não afetaram a produção e a qualidade de embriões.

Palavras-chaves: hormônios gonadotróficos, reprodução animal, superovulação, transferência de embrião.

\section{SUMMARY}

This study aimed to investigate the efficiency and standards in the superrovulatory response to products with different relationships FSH:LH. It Were used 43 female Boer goats breed. On a random day of the estrous cycle, the animals received half of an implant of progesterone $\left(\right.$ Crestar $\left.^{\circledR}\right)$. In the eleventh day, began stimulation to superovulation through the use of FSH fractionated in six decreasing doses administered every 12 hours and the animals were separated into two groups: group I (GI, $\mathrm{n}=22$ ) received $200 \mathrm{mg}$ of $\mathrm{FSH}$ (Folltropin ${ }^{\circledR}$ ) intramuscularly (i.m) on days D11 $(62,5 \mathrm{mg})$, D12 (35,0mg) and D13 (2,5mg), while the group II (GII, $\mathrm{n}=21$ ) received 300UI FSH (Pluset $^{\circledR}$ ) intramuscularly on days D11 (75UI), D12 (50UI) and D13 (25UI). In 60 and 72h after onset of superovulation the implants were removed and applied two doses of $125 \mu \mathrm{g}$ de Cloprostenol $\left(\mathrm{Ciosin}^{\circledR}\right)$ by intravulvosubmucosal 
in all animals. Twelve, 24 and 36 hours after removal of the implant, the females were placed with four breeders to be covered. In D16, the implants were reinserted and 24 hours before collection of embryos the implants were withdrawl and administered $125 \mu \mathrm{g}$ de Cloprostenol in each animal intramuscularly. Embryo were collected on D21, selected and classified. The results obtained in this study showed no statistical difference between groups regarding the number of recovered structures, viable embryos, degenerate embryos and unfertilized structures. So, the different relationships FSH:LH evaluated did not affect embryos production and quality.

Keyswords: animal reproduction, gonadotropic hormones, superovulation, embryo transfer.

\section{INTRODUÇÃO}

A transferência de embriões (TE) em pequenos ruminantes é uma ferramenta que têm ganhado importância (ANDROUKOVITCH et al., 2002), apesar da variabilidade de resultados mostrar-se ainda limitadora quanto a difusão. Atribuem-se tais limitações às atividades que envolvem desde seleção de animais (GONZÁLEZ-BULNES et al., 2004), técnica de superovulação (COGNIÉ et al., 2003) e método de coleta utilizado (GUSMÃO et al., 2003).

Os resultados imprevisíveis, associados a altos custos, restringem o uso em larga escala de programas de superovulação em caprinos (BALDASSARE \& KARATZAS, 2004). Outro fator considerável é a qualidade dos embriões que é influenciada pelo tipo e quantidade de gonadotrofinas utilizadas (IHEUKWUMERE, 2008).

No que diz respeito à seleção dos animais, já se sabe que fatores intrínsecos como raça, idade, individualidade, status ovariano, estádio de lactação e fatores extrínsecos como nutrição, saúde e época do ano são responsáveis pela variabilidade das respostas à superovulação (COGNIÉ et al., 2003; GONZÁLEZ-BULNES et al., 2004).

Nesse sentido, tem-se buscado a adequação de diferentes protocolos, ou fármacos que os compõem, dos quais os mais pesquisados são, a saber: o hormônio folículo estimulante de ovino (oFSH) ou de suíno (pFSH) e Gonadotrofina coriônica equina (eCG) ou humana (hCG), no intuito de tornar a TE nessa espécie mais eficiente (IHEUKWUMERE, 2008).

Os produtos comerciais de FSH utilizam diferentes proporções FSH:LH. O Pluset ${ }^{\circledR}$ apresenta uma relação FSH e LH próxima de 1:1, enquanto $\mathrm{o}$ Folltropin $^{\circledR}$ apresenta baixa concentração de LH, com uma relação FSH:LH de 5,25:1 (KELLY et al., 1997). Segundo esses autores, os efeitos de altas concentrações de LH incluem elevado número de folículos anovulatórios, ocorrência de embriões degenerados, aumento exacerbado do estroma ovariano, demora no retorno à ciclicidade e baixa margem de segurança relacionada à dose total.

As gonadotrofinas hipofisárias são capazes de aumentar os níveis de anticorpos desde que sua obtenção tenha sido de uma espécie diferente daquela nas quais serão utilizadas (SIMPLÍCIO et al., 2002). Todavia, esses resultados não são consensuais (ANDRIOLI et al., 1999; BARI et al., 2001). Convém lembrar que há a necessidade de múltiplas aplicações do FSH para estimular o crescimento folicular e ovulações (COGNIÉ \& BARIL, 2002).

Assim, este trabalho objetivou averiguar a eficiência e padrões na resposta superovulatória em caprinos da raça Boer, submetidos a produtos com diferentes relações FSH:LH. 


\section{MATERIAL E MÉTODOS}

O experimento foi realizado na fazenda Santa Fé, situada no município de Iaçú, na região semiárida do Estado da Bahia (longitude oeste $40^{\circ} 26^{\prime}$ e latitude sul $12^{\circ} 33^{\prime}$ ) durante os meses de maio a novembro de 2009.

Para realização do estudo, foram utilizadas 43 fêmeas caprinas da raça Boer, multíparas de 3 a 4 anos de idade, com peso entre 50 e 60 quilos. Os animais foram mantidos em sistema de criação semi-intensivo, com alimentação à base de capim Buffel (Cenchrus ciliaris), suplementado com $300 \mathrm{~g}$ de ração comercial, composta de $18 \%$ de proteína bruta. As refeições ocorriam duas vezes ao dia e lhes eram fornecidos água e mineralização (Caprinofós ${ }^{\circledR}$, Tortuga, São Paulo, Brasil) ad libitum.

As doadoras receberam em um dia aleatório do ciclo estral, considerado dia zero (D0), metade de um implante auricular de progesterona que corresponde a $1,5 \mathrm{mg}$ de Norgestomet (Crestar $^{\circledR}$, Shering-Plough, São Paulo, Brasil). No dia 11 do protocolo, os animais foram separados em dois grupos, nos quais se iniciou o estímulo à superovulação com o emprego do FSH, aplicado por 3 dias consecutivos (D11 a D13), fracionados em seis doses decrescentes, administradas a cada 12 horas.

Cada animal do grupo I (GI, $n=22)$ recebeu 200mg de FSH (Folltropin ${ }^{\circledR}$, Bioniche Animal Health, Toronto, Canadá) aplicados por via intramuscular (i.m.) nos dias D11 (62,5mg), D12 (35,0mg) e D13 (2,5mg). O grupo II (GII, $\mathrm{n}=21$ ) recebeu 300UI de $\mathrm{FSH}$ (Pluset $^{\circledR}$, Calier, IF Serono, Itália) i.m. aplicados nos dias D11 (75UI), D12 (50UI) e D13 (25UI).

Todos os animais receberam duas doses de $125 \mu \mathrm{g}$ de Cloprostenol $\left(\operatorname{Ciosin}^{\circledR}\right.$,
Schering-Plough, São Paulo, Brasil) pela via intravulvosubmucosa, 60 e $72 \mathrm{~h}$ após o início da superovulação e, quando da última administração hormonal, os implantes de progesterona foram removidos, identificados e armazenados individualmente sob refrigeração. Doze, 24 e 36 horas após, as fêmeas foram levadas aos reprodutores, comprovadamente férteis, para observação de estro e cobertura. Para tal, foram utilizados quatro reprodutores da raça Boer, com idade entre 3 a 4 anos, mantidos em sistema intensivo de criação, alimentados com feno de capim tifton (Cynodon spp) $500 \mathrm{~g}$ de ração comercial, composta de $18 \%$ de proteína bruta. No D16, pela manhã, os implantes foram reinseridos nas respectivas fêmeas para prevenir a regressão prematura dos corpos lúteos.

Nas 24h anteriores a coleta dos embriões, as doadoras foram mantidas em jejum hídrico e sólido e, $12 \mathrm{~h}$ précoleta, os implantes foram retirados e administrou-se $125 \mu \mathrm{g}$ de Cloprostenol i.m. para cada animal. Os embriões foram coletados no D21 pelo método transcervical. Empregou-se metodologia e sistema fechado descritos por Gusmão et al. (2002), isto é, utilizou-se como meio o PBS (Phosphate Buffered Saline - Nutricell - Brasil) na quantidade de $500 \mathrm{~mL}$ por animal e sonda NelatonRobinson (Rusch Inc - Alemanha) $n^{\circ}$ 10 ou 12. Posteriormente, os embriões foram selecionados e classificados segundo o Manual da Sociedade Internacional de Transferência de Embriões - IETS (STRINGFELLOW \& SEIDEL, 1999). Os dados foram processados pelo Statistical Package for Social Science, versão 19 (SPSS, 2010) e compararam-se as médias entre os tratamentos pelo teste t de Student para as seguintes variáveis: número de estruturas, embriões viáveis, embriões 
degenerados, estruturas não fertilizadas e qualidade dos embriões.

\section{RESULTADOS E DISCUSSÃO}

A passagem da sonda pela cérvix foi possível em $100 \%$ das doadoras, resultado superior aos encontrados por Andrioli et al. (1999) e Lima-Verde et al. (2003), os quais foram, respectivamente, de $73,3 \%$ e $95,0 \%$. Deve-se ressaltar que esses autores utilizaram cabras da raça Saanen em seus estudos, que podem apresentar intrinsecamente uma menor taxa de transposição cervical. Já Androukovitch et al. (2002), quando trabalharam com cabras da raça Boer, conseguiram uma taxa de transposição cervical de 83,3\%, também inferior aos resultados encontrados no presente estudo, o que pode ser justificada pelo fato do autor ter usado fêmeas jovens em seu experimento, enquanto que neste trabalho foram priorizadas fêmeas multíparas que, provavelmente, apresentaram um maior diâmetro do lume cervical.

As primeiras colheitas de embriões, por meio da técnica transcervical, em caprinos registraram taxa de recuperação do líquido infundido intrauterinamente de 90\% (BONDURANT et al., 1984). Resultados semelhantes foram reportados por Androukovitch et al. (2002) e Silva et al. (2010) os quais recuperaram $94,3 \%$ e 90,3\% do líquido infundido em animais da raça Boer, ao utilizarem sonda uretral humana.

Esses resultados corroboram com os dados deste experimento: $96,8 \%$ de recuperação do meio infundido. Entretanto, os valores obtidos foram superiores aos encontrados por Melican \& Gavin (2008) que, ao usarem cateter uretral em fêmeas da raça Saanen, Alpina e Toggenburg obtiveram recuperação de $80 \%$ do líquido infundido.

O tempo de coleta no presente experimento foi de 29,08 \pm 9,2 minutos. Para essa medição, os resultados encontrados na literatura variam entre 18,43 \pm 4,0 minutos (ANDRIOLI et al., 1999) e 30 a 60 minutos (MELICAN \& GAVIN, 2008). Possivelmente, tal variação deve-se a fatores como raça, ordem de partos, aplicação de drogas para facilitar a passagem da sonda, ou mesmo, fatores inerentes ao indivíduo (ALMEIDA et al., 2002; GUSMÃO et al., 2009; LEHLOENYA \& GREYLING, 2010).

Neste experimento, ao comparar produtos com diferentes relações FSH:LH (Folltropin ${ }^{\circledR}$ vs Pluset $^{\circledR}$ ), obtidos da hipófise de suínos, não houve diferença estatística quanto ao número de estruturas recuperadas, embriões viáveis, embriões degenerados e estruturas não fertilizadas (Tabela 1). Isso torna esta pesquisa consonante com diversos estudos efetuados sobre a espécie bovina, nos quais também não foi encontrada diferença estatística em relação a essas características em vacas superovuladas com os produtos supracitados (NASCIMENTO et al., 2004; ALVAREZ et al., 2006).

Dessa forma, os resultados do presente estudo, associados às demais investigações citadas, comprovam que tanto Folltropin ${ }^{\circledR}$ quanto Pluset ${ }^{\circledR}$ podem ser eficientes para superovulação de fêmeas das espécies bovina e caprina. Uma possível explicação para a apresentação desses dados se constitui na existência de um número limitado de receptores de FSH nas células da granulosa dos folículos de ambas as espécies e no aumento do grau de pureza do fármaco, acima de dado nível, o qual pode não exercer nenhum efeito sobre a resposta superovulatória (ALVAREZ et al., 2006). 
Segundo Magalhães et al. (2009), as respostas aos protocolos de superovulação dependem da base farmacológica, da fonte utilizada para processamento desse hormônio e grau de pureza. A utilização de FSH processados, a partir da hipófise de ovinos ou suínos, não mostrou diferença quanto à recuperação dos embriões, no entanto, ao se comparar o uso de Gonadotrofina menopáusica humana (hMG), composição hormonal com relação FSH:LH de 1:1, com pFSH $\left(\right.$ Folltropin $\left.^{\circledR}\right), \quad$ foram encontradas variações significativas nas taxas de ovulação e número de embriões recuperados ou viáveis em caprinos (IHEUKWUMERE, 2008).

Tabela 1. Média e desvio padrão das estruturas obtidas na superovulação de cabras Boer com Folltropin ${ }^{\circledR}$ e Pluset ${ }^{\circledR}$

\begin{tabular}{lcccc}
\hline Tratamento & $\begin{array}{c}\text { Estruturas } \\
\text { totais }\end{array}$ & $\begin{array}{c}\text { Embriões } \\
\text { viáveis }\end{array}$ & $\begin{array}{c}\text { Embriões } \\
\text { degenerados }\end{array}$ & $\begin{array}{c}\text { Estruturas não } \\
\text { fertilizadas }\end{array}$ \\
\hline Folltropin & $6,18 \pm 6,36$ & $4,41 \pm 4,38$ & $1,12 \pm 2,64$ & $1,65 \pm 2,37$ \\
Pluset & $6,63 \pm 4,44$ & $4,31 \pm 4,09$ & $0,56 \pm 0,89$ & $1,88 \pm 3,64$ \\
\hline Total & $6,39 \pm 5,43$ & $4,37 \pm 4,20$ & $0,85 \pm 1,98$ & $1,76 \pm 3,01$ \\
\hline
\end{tabular}

Iheukwumere (2008) comparou o uso de pFSH, oFSH e hMG na superovulação de cabras e não encontrou disparidade para o número de embriões recuperados, degenerados e estruturas não fertilizadas entre o pFSH e oFSH. Deve-se fazer uma ressalva, quanto ao oFSH: este se apresentou superior às outras duas drogas em relação ao número de embriões fertilizados e transferíveis. Na ocasião, o resultado apresentado para embriões recuperados que usavam $\mathrm{pFSH}$ foi de 7,25 embriões por fêmea, superior ao encontrado neste trabalho (4,37 embriões por fêmea). Esses resultados justificam-se devido às diferentes técnicas de coleta embrionária utilizadas, cirúrgica ou transcervical, pois, segundo Silva et al. (2003), a técnica de coleta pelo método cirúrgico apresenta índices de recuperação superiores à técnica transcervical.

Os tratamentos não interferiram no número médio de embriões produzidos de acordo com a qualidade (Tabela 2). Esse resultado diverge do encontrado por Iheukwumere (2008), que obteve média de embriões com qualidade 1 (excelente ou bom) e qualidade 2 (regular), respectivamente, de 3,00 e 1,00 (LINDNER \& WRIGHT, 1983), ao utilizar Folltropin ${ }^{\circledR}$. Tais dados podem ser explicados pela característica racial das doadoras caprinas participantes dos experimentos, visto que Iheukwumere (2008) trabalhou com doadoras da raça West African Dwarf, fator citado por vários autores como responsável pela variabilidade das respostas aos tratamentos superovulatórios (ALMEIDA et al., 2002; COGNIÉ et al., 2003; GONZÁLEZBULNES et al., 2004).

Ao se comparar os achados desse estudo aos de Silva et. al. (2010), utilizando Pluset $^{\circledR}$ em programas de superovulação em fêmeas Boer multíparas verificaram uma superioridade nas médias de embriões transferíveis com qualidade 1 $(5,3)$ e $2(2,15)$ que comprova a influência da resposta individual no sucesso dos tratamentos de superovulação em cabras. 
Rev. Bras. Saúde Prod. Anim., Salvador, v.13, n.4, p.1066-1073 out./dez., 2012 http://www.rbspa.ufba.br ISSN 15199940

Tabela 2. Média e desvio padrão do número de embriões produzidos de acordo com a qualidade, em doadoras caprinas superovuladas com Folltropin ${ }^{\circledR}$ e Pluset $^{\circledR}$

\begin{tabular}{lcccc}
\hline Tratamento & Embriões viáveis & Qualidade 1 & Qualidade 2 & Qualidade 3 \\
\hline Folltropin & $4,41 \pm 4,38$ & $1,68 \pm 2,69$ & $0,86 \pm 0,94$ & $2,14 \pm 3,06$ \\
Pluset & $4,31 \pm 4,09$ & $1,71 \pm 2,26$ & $1,38 \pm 1,98$ & $1,62 \pm 1,74$ \\
\hline Total & $4,37 \pm 4,20$ & $1,70 \pm 2,46$ & $1,12 \pm 1,54$ & $1,88 \pm 2,49$ \\
\hline
\end{tabular}

Qualidade 1 - excelente ou bom; 2 - regular; 3 - pobre.

Concluiu-se que as drogas superovulatórias com diferentes relações FSH:LH avaliadas não afetaram a produção e a qualidade de embriões, o que sugere que ambos os fármacos são eficazes e podem ser usados em protocolos de superovulação em caprinos da raça Boer.

\section{REFERÊNCIAS}

ALMEIDA, V.M.; CÂMARA, D.R.; SALLES, H.O. Colheita de embriões via transcervical em ovinos. Revista Brasileira de Reprodução Animal, v.26, n.5 p.82-84, 2002.

ALVAREZ, R.H.; PIRES. R.M.L.; MARTINEZ, A.C. Resposta ovariana e produção de embriões de vacas superovuladas com Pluset ou Folltropin em dose única subcutânea. Acta

Scientiae Veterinariae, v.34, p.516, 2006. Supl. 1.

ANDRIOLI, A.; SIMPLÍCIO, A.A.; SOARES, A.T.; VISINTIN, J.A.

Eficiência da recuperação de embriões e os efeitos de consecutivas colheitas sobre o aparelho reprodutor de doadoras da espécie caprina. Brazilian Journal of Veterinary Research and Animal Science, v.36, n.3, 1999. Disponivel em: $<$ http://www.scielo.br/scielo.php?script=sc i_arttext\&pid=S141395961999000300006>. Acesso em: 20 out. 2011.
ANDROUKOVITCH, J.L.; KOZICKI, L.E.; KOZEMJAKIM, D.A.; ABREU, R.M.; NUNEZ, C. Coleta de embriões caprinos com sonda uretral humana. Archives of Veterinary Science, v.7, n. 1, p.37-41, 2002.

BALDASSARE, H.; KARATZAS, C.N. Advanced assisted reproduction technologies (ART) in goats. Animal Reproduction Science, v.82-83, p.255266, 2004.

BARI, F.; KHALID, M.; WOLF, B.; HARESIGN, W.; MURRAY, A.; MERRELL, B. The repeatability of superovulatory response and embryo recovery in sheep. Theriogenology, v.56, n.1, p.147-155, 2001.

BONDURANT, R.H.; SKIRROW, S.; ANDERSON, G.B.; HANSON, F.; ROGERS, W.H. Nonsurgical collection of blastocysts from dairy goats.

Theriogenology, v.22, n.4, p.423-431, 1984.

COGNIÉ, Y.; BARIL, G. Le point sur la production et le transfert d'embryons obtenus in vivo e in vitro chez la bebris e la chèvre. Productions Animales, v.15, p.199-207, 2002.

COGNIÉ, Y.; BARIL, G.; POULIN, N.; MERMILLOD, P. Current status of embryo technologies in sheep and goat. Theriogenology, v.59, n.1, p.171-188, 2003. 
GONZÁLEZ-BULNES, A.; BAIRD, D.T.; CAMPBELL, B.K.; COCERO, M.J.; GARCÍA-GARCÍA, R.M.; KEITH INSKEEP, E.; LÓPESSEBASTIÁN, A.; McNEILLY, A.S.; SANTIAGO-MORENO, J.; SOUZA, C.J.H.; VEIGA-LÓPES, A. Multiple factors affecting the efficiency of multiple ovulation and embryo transfer in sheep and goats.

Reproduction, Fertility and Development, v.16, n.4, p.421-435, 2004.

GUSMÃO, A.L.; RESENDE, J.; OLIVEIRA, J.V.L.; RIBEIRO FILHO, A.L. MOURA, J.C.A. Modificação da técnica de colheita transcervical de embriões de cabras com um cateter disprovido de balão. Revista

Brasileira de Reprodução Animal, v.26, p.101-103, 2002. Supl. 5.

GUSMÃO, A.L.; MOURA, J.C.A.; CHALHOUB, M.; RIBEIRO FILHO, A.L. Colheita, avaliação e criopreservação de embriões caprinos. Revista Brasileira de Reprodução Animal, v.27, n.2, p.115-119, 2003.

GUSMÃO, A.L.; SILVA, J.C.; BITTENCOURT, T.C.C.; MARTINS, L.E.P. GORDIANO, H.D.; BARBOSA, L.P. Coleta transcervical de embriões em ovinos da raça Dorper no semi-árido do nordeste brasileiro. Arquivo Brasileiro de Medicina Veterinária e Zootecnia, v.61, p.313318, 2009.

IHEUKWUMERE, F. The effects of different gonadotrophin treatments on the embryo generation and quality of embryos in west african dwarf goats. Journal of Agriculture and Social Research, v.8, n.1, p.57, 2008.
KELLY, P.; DUFFY, P.; ROCHE, J.F.; BOLAND, M.P. Superovulation in cattle: effect of FSH type and method of administration on follicular growth, ovulatory response and endocrine patterns. Animal Reproduction Science, v.46, p.1-14, 1997.

LEHLOENYA, K.C.; GREYLING, J.P.C. The ovarian response and embryo recovery rate in Boer Goat does following different superovulation protocols, during the breeding season. Small Ruminant Research, v.88, p.3843, 2010.

LIDNER, G.M.; WRIGHT JUNIOR, R.W. Bovine embryo morphology and evaluation.Theriogenology, v.20, p.407-416, 1983.

LIMA-VERDE, J.B.; LOPES JÚNIOR, E.S.; TEIXEIRA, D.I.A.; PAULA, N.R.O.; MEDEIROS, A.A.; ARRUDA, I.J.; CÃMARA, A.C.;RONDINA, D.; FREITAS, V.J.F. Colheita de embriões pela técnica transcervical em cabras da raça Saanen criadas nos trópicos.

Revista Brasileira de Reprodução Animal, v.27, n.3, p.489-490, 2003.

MAGALHÃES, D.M.; FERNANDES, D.D.; ARAUJO, V.R., ALMEIDA, A.P.; MATOS, M.H.T.; FIGUEIREDO, J.R. Papel do hormônio folículo estimulante na foliculogênese in vitro e in vitro. Revista Brasileira de Reprodução Animal [Online], v.33, n.4, p.171-182, 2009.

MELICAN, D.; GAVIN, W. Repeat superovulation, non-surgical embryo recovery, and surgical embryo transfer in trangenic dairy goats.

Theriogenology, v.69, p.197-203, 2008. 
NASCIMENTOS, A.B.; SENEDA, M.M.; SILVEIRA, E.C.; PANSARD, H. Associação de gonadotrofinas para a superovulação de vacas doadoras de embriões. Ciência Animal, v.14, p.8994, 2004.

SILVA, M.H.; MEDEIROS, L.R.D.; GOMES NETO, O.C.; PROCÓPIO, C.S.; CÂMARA, D.R.; SILVEIRA FILHO, M.E.M.; CARNEIRO, G.F. Recuperação e viabilidade embrionária pelos métodos cirúrgico e transcervical em programa comercial de transferência de embriões em caprinos da raça Boer. Acta Scientiae Veterinariae, v.31, p.582, 2003. Supl.1.

SILVA, S.V.; CARNEIRO, G.F.; GUERRA, M.M.P.; GOMES NETO, O.C.; BATISTA, A.M.; WISCHRAL, A. Efeito das condições reprodutivas e climáticas na produção de embriões de cabras Boer superovuladas. Ciência Animal Brasileira, v.11, n.3, p.570, 2010.
SIMPLÍCIO, A.A.; SALLES, H.O.; SANTOS, D.O. Transferência de embriões nos pequenos ruminantes domésticos. Revista Brasileira de Reprodução Animal, v.1, p.17-22, 2002. Supl. 5.

SPSS Inc. Statistical Analysis Using SPSS. Version 19. Chicago, 2010.

STRINGFELLOW, D.A; SEIDEL, S.M. Manual da Sociedade Internacional de Transferência de Embriões. 3.ed. São Paulo: Sociedade Brasileira de Transferência de Embriões, 1999. 180p.

Data de recebimento: 12/01/2012

Data de aprovação: 21/09/2012 